\title{
Critical size limits for collinear and spin-spiral magnetism in $\mathrm{CoCr}_{2} \mathrm{O}_{4}$
}

\author{
D. Zákutná $\odot,{ }^{1,2}$ A. Alemayehu, ${ }^{3}$ J. Vlček ${ }^{4}$ K. Nemkovski $\odot,{ }^{5}$ C. P. Grams, ${ }^{6}$ D. Nižňanský, ${ }^{3, *}$ \\ D. Honecker ${ }^{0},{ }^{1, \dagger}$ and S. Disch $\mathbb{1}^{2, \dagger}$ \\ ${ }^{1}$ Institut Laue-Langevin, 71 Avenue des Martyrs, F-38042 Grenoble, France \\ ${ }^{2}$ Department für Chemie, Universität zu Köln, Luxemburger Strasse 116, 50939 Köln, Germany \\ ${ }^{3}$ Department of Inorganic Chemistry, Faculty of Science, Charles University in Prague, Hlavova 2030/8, 12843 Prague 2, Czech Republic \\ ${ }^{4}$ Department of Physics and Measurements, University of Chemistry and Technology Prague, Technicka 5, 16628 Prague, Czech Republic \\ ${ }^{5}$ Forschungszentrum Jülich GmbH, Jülich Centre for Neutron Science (JCNS) at Heinz Maier-Leibnitz,Zentrum (MLZ), \\ Lichtenbergstrasse 1, 85748 Garching, Germany \\ ${ }^{6}$ II. Physikalisches Institut, Universität zu Köln, Zülpicher Strasse 77, 50937, Köln, Germany
}

(Received 29 August 2019; published 27 November 2019)

\begin{abstract}
The multiferroic behavior of $\mathrm{CoCr}_{2} \mathrm{O}_{4}$ results from the appearance of conical spin-spiral magnetic ordering, which induces electric polarization. The magnetic ground state has a complex size-dependent behavior, which collapses when reaching a critical particle size. Here the magnetic phase stability of $\mathrm{CoCr}_{2} \mathrm{O}_{4}$ in the size range of $3.6-14.0 \mathrm{~nm}$ is presented in detail using the combination of neutron diffraction with $\mathrm{XYZ}$ polarization analysis and macroscopic magnetization measurements. We establish critical coherent domain sizes for the formation of the spin spiral and ferrimagnetic structure and reveal the evolution of the incommensurate spin spiral vector with particle size. We further confirm the presence of ferroelectric polarization in the spin spiral phase for nanocrystalline $\mathrm{CoCr}_{2} \mathrm{O}_{4}$.
\end{abstract}

DOI: 10.1103/PhysRevB.100.184427

\section{INTRODUCTION}

The coupling of two ferroic properties, known as multiferroism is widely studied due to its applicability in spintronics [1]. Many materials, such as $\mathrm{RMnO}_{3}$ and $\mathrm{RMn}_{2} \mathrm{O}_{5}(\mathrm{R}=$ $\mathrm{Dy}, \mathrm{Tb}$, or $\mathrm{Ho}$ ) have ferroelectric properties emerging in an antiferromagnetic state [2-5]. Multiferroics, for which the ferroelectricity arises from a spiral magnetic structure are, in general, considered as ferroelectrics showing the strongest sensitivity to magnetic field [6]. In the case of $\mathrm{CoCr}_{2} \mathrm{O}_{4}$, ferroelectric polarization arises from the spin-spiral structure, which is formed due to the strong direct exchange interaction in the $\mathrm{B}$ spinel site [7]. Thus, $\mathrm{CoCr}_{2} \mathrm{O}_{4}$ is a multiferroic material with both spontaneous magnetization and electric polarization of spin spiral origin[8-10]. The magnetic properties of nanocrystalline $\mathrm{CoCr}_{2} \mathrm{O}_{4}$ change significantly from its bulk counterpart, for instance, no lock-in transition $T_{\text {lock }}$ to the ground state is found [11-19]. Moreover, it is difficult to deduce clearly the spin spiral transition temperature $T_{\mathrm{s}}$ and the presence of the associated spin spiral magnetic ordering from volume-averaged magnetization measurements [11]. Few studies employed polarized neutron diffraction measurements, where the appearance of magnetic satellite reflections is a clear indicator of the spin spiral magnetic ordering in $\mathrm{CoCr}_{2} \mathrm{O}_{4}[13,18,19]$ and $\mathrm{Fe}$-doped $\mathrm{CoCr}_{2} \mathrm{O}_{4}$ [20]. A strong influence of the particle size on the magnetic properties is

\footnotetext{
${ }^{*}$ Deceased

${ }^{\dagger}$ Now at: Physics and Materials Science Research Unit, University of Luxembourg, 162A Avenue de la Faïencerie, L-1511 Luxembourg, Grand Duchy of Luxembourg.

ॠCorresponding author: sabrina.disch@uni-koeln.de
}

suggested [13]: nanoparticles (NPs) with an average grain size of $22 \mathrm{~nm}$ reveal a clear spin spiral configuration below $T_{\mathrm{s}}=$ $27 \mathrm{~K}$, similar to the bulk material, whereas small NPs of 3-nm size exhibit a collective cluster glass behavior.

Here we present a detailed magnetic phase diagram of $\mathrm{CoCr}_{2} \mathrm{O}_{4}$ for the size range of $3.6-14.0 \mathrm{~nm}$ obtained by combining polarized neutron diffraction with macroscopic magnetization measurements. The grain-size and temperature-dependent boundaries of the magnetic phases are deduced from the fundamental reflections for collinear magnetic ordering and magnetic satellites of spin spiral ordering. Next to the critical size limits for both collinear and spin spiral magnetism, we confirm multiferroic properties in the spin spiral phase through ferroelectric polarization.

\section{EXPERIMENT}

Cobalt chromite NPs were synthesized by a hydrothermal method according to [11]. An aqueous solution of chromium and cobalt chloride hexahydrate in a molar ratio $\left(\mathrm{Cr}^{3+}: \mathrm{Co}^{2+}=2: 1\right)$ was combined with a water/ethanol $(1: 1)$ solution of sodium oleate. The final mixture was transferred into an autoclave with 50-mL teflon liner and heated to $200^{\circ} \mathrm{C}$ for $16 \mathrm{~h}$. After cooling down, the liquid phases were discarded, and the sedimented nanoparticles were purified three times by dispersing in hexane and precipitating in ethanol to remove all remaining free oleic acid and sodium chloride. The six different particle sizes were obtained by annealing the amorphous as-prepared nanoparticles at 300, 350, 400, 450, 500 , and $550^{\circ} \mathrm{C}$ for $2 \mathrm{~h}$. In the following, the samples are labeled with the prefix AA and numbers corresponding to the annealing temperature. 
Powder x-ray diffraction (PXRD) was performed using a PANalytical X'Pert PRO diffractometer with $\mathrm{Cu} \mathrm{K}_{\alpha}$ radiation $(\lambda=1.54 \AA)$. The diffractometer is equipped with a PIXcel detector and a secondary monochromator. The samples were measured in the $2 \theta$-range of $5-80^{\circ}$ with a step size of $0.003^{\circ}$. The instrumental broadening was obtained with a $\mathrm{LaB}_{6}$ reference (SR 660b, NIST). For the Le-Bail refinement of the diffraction patterns the FULLPROF software [21] was used.

Transmission electron microscopy (TEM) was done in a bright-field (BF) mode on a ZEISS LEO 902 microscope with a $\mathrm{LaB}_{6}$ cathode operating at $120 \mathrm{kV}$. The samples were deposited onto a coated copper grid. The mean particle diameter was statistically determined from at least 200 particles in different BF images and fitted with log-normal size distribution.

Integral magnetization measurements were performed on a Quantum Design Physical Property Measurement System (PPMS) equipped with a superconducting magnet and a vibrating sample magnetometer (VSM) option. The zero-field-cooled (ZFC) and field-cooled (FC) magnetization measurements were done between 2 and $300 \mathrm{~K}$ with a heating rate of $1 \mathrm{~K} / \mathrm{min}$ in an applied magnetic field of $\mu_{0} H=10 \mathrm{mT}$. The isothermal, field-dependent magnetization was measured at the base temperature of $2,10,30$, and above $80 \mathrm{~K}$. AC susceptometry was performed at the Quantum Design Physical Property Measurement System (PPMS Evercool) equipped with a superconducting magnet and an ACMSII option. The temperature dependence of the AC field response was determined with AC field amplitude of $0.7 \mathrm{mT}$ at frequencies of 10 , 95, 292, and $900 \mathrm{~Hz}$.

Polarized neutron scattering experiments with XYZ polarization analysis were performed at the DNS instrument $[22,23]$ at MLZ in Garching, Germany. Measurements covered a temperature range of $3.2-100 \mathrm{~K}$ using a neutron wavelength of $\lambda=4.2 \AA$. $\mathrm{NiCr}$ and vanadium were used as references for the correction of flipping ratio and detector efficiency, respectively. The magnetic, nuclear coherent and spin-incoherent contributions were separated from the total scattering cross section by means of XYZ-polarization analysis using the "DNSPLOT" interface [24].

Measurements of the macroscopic polarization of $\mathrm{CoCr}_{2} \mathrm{O}_{4}$ nanoparticles were performed on a separate part of the sample batches that was slightly pressed and put between capacitor plates. Due to the random orientation of the particles a direct measurement of the polarization is not expected to yield a large signal [25]. Therefore, we applied a static electric field $E_{\mathrm{dc}} \approx 1 \mathrm{kV} / \mathrm{mm}$ at temperatures above the expected phase transition and cooled the sample down to $10 \mathrm{~K}$. To orient the particles, we applied the magnetic field perpendicular to $E_{\mathrm{dc}}$ that was oscillated between 0.1 and $-0.1 \mathrm{~T}$ at this temperature. After this poling procedure, the electric field was turned off, the sample reheated to $40 \mathrm{~K}$, and the actual temperature-dependent measurement was performed on cooling in zero magnetic field to find $T_{\mathrm{s}}$.

\section{RESULTS AND DISCUSSION}

\section{A. Morphology and structure}

Cobalt chromite NPs with tunable particle size and good crystallinity were obtained by temperature-dependent

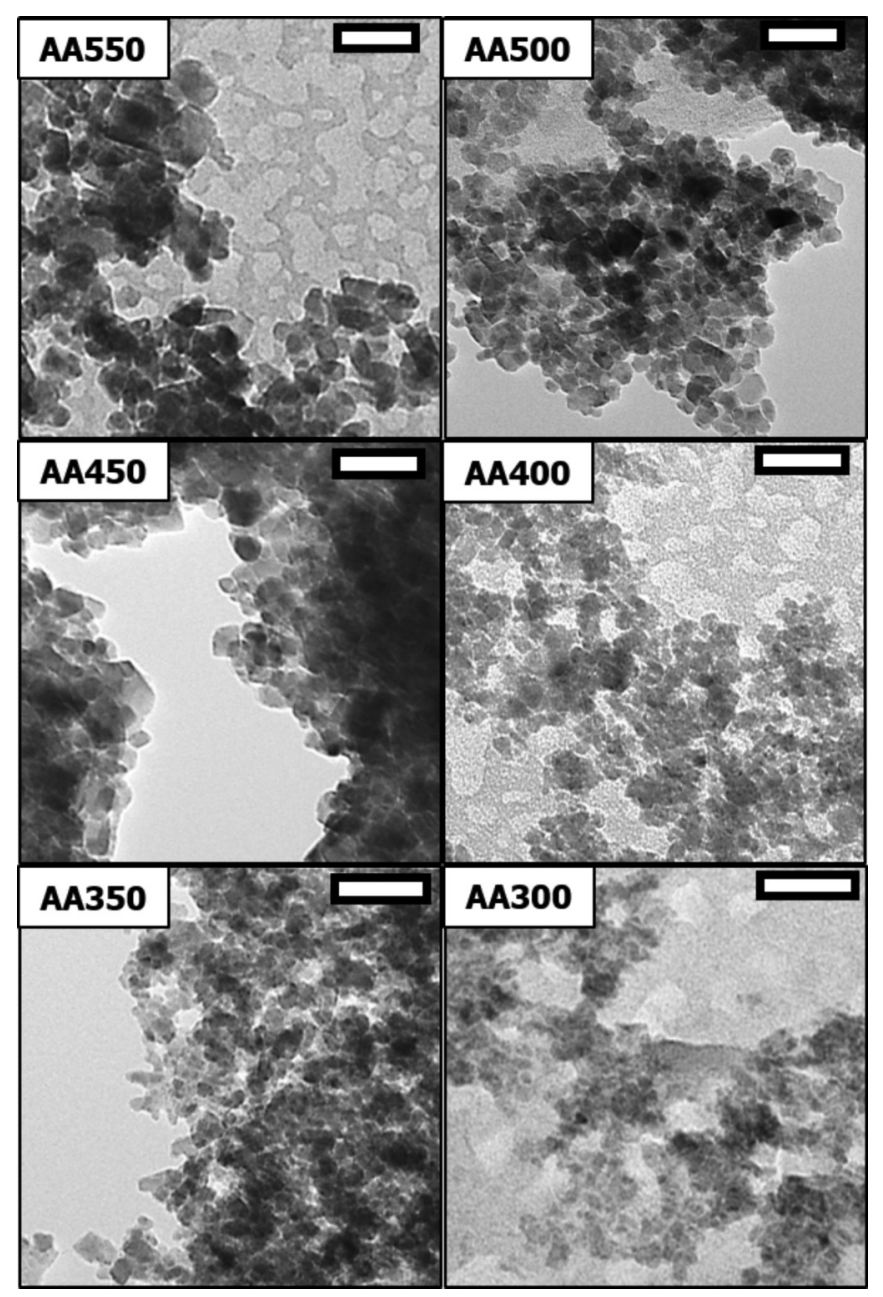

FIG. 1. BF TEM micrographs of cobalt chromite samples (scale bars: $50 \mathrm{~nm})$.

annealing of the amorphous $\mathrm{CoCr}_{2} \mathrm{O}_{4}$ precursor gained from hydrothermal synthesis (for more information see [26]). The cobalt chromite NPs have nearly spherical morphology as confirmed by TEM (Figs. 1 and S1 [26]). The samples are labeled as AA (annealing temperature in ${ }^{\circ} \mathrm{C}$ ). PXRD data (Fig. S2, Table SI) [26] were refined according to the spinel structure of $\mathrm{CoCr}_{2} \mathrm{O}_{4}(F d \overline{3} m$ space group) using Le-Bail analysis, and confirm phase purity for all samples. A small impurity of $\mathrm{NaCl}$ was detected for the sample AA350 and removed before the neutron scattering experiment by a purification step. The structurally coherent particle sizes from PXRD are in good general agreement, but slightly smaller than the particle sizes obtained by TEM analysis (Fig. S3 [26]) indicating structural disorder near the particle surface. The size distribution of the NPs is reasonably narrow and in the range of $\sigma_{\log }=0.1-0.2$ (Table SII [26]). The nanoparticle size increases with annealing temperature, and simultaneously the lattice parameter slowly approaches the bulk value (Fig. S3 [26]).

\section{B. Magnetic properties}

The FC and ZFC magnetization measurements [Figs. 2(a) and 2(b)] reveal three distinct magnetic phase transitions. The Curie temperature $\theta_{\mathrm{C}}$ is determined by linear extrapolation 

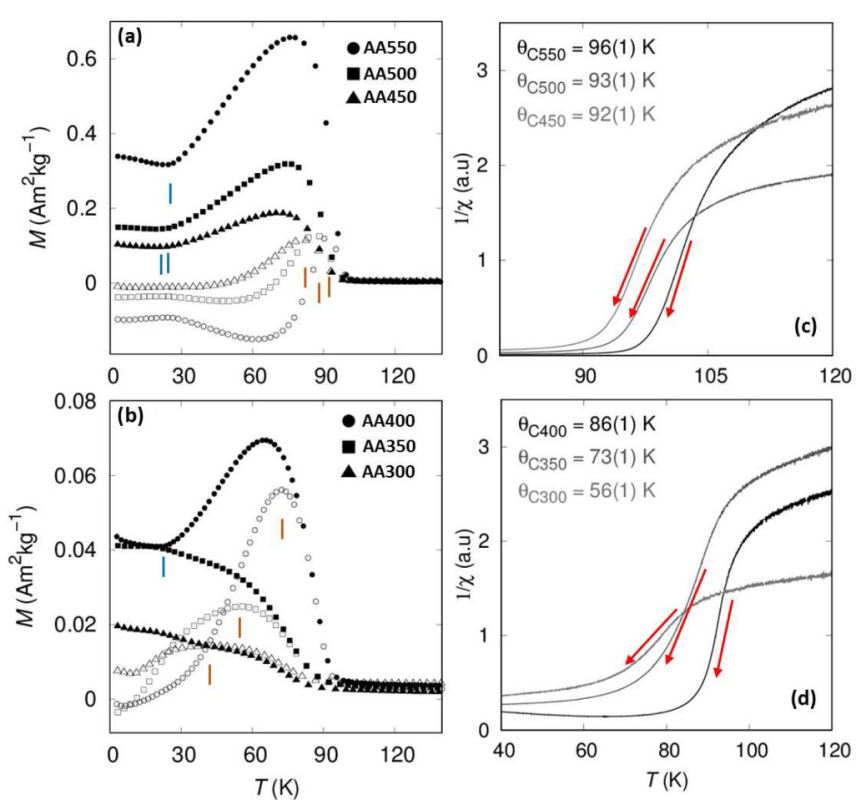

FIG. 2. (a-b) ZFC/FC (full/open symbols) magnetization measurements of cobalt chromite NPs with different particle sizes. Blue and brown vertical lines correspond to the transition temperatures $T_{\mathrm{s}}$ and $T_{\mathrm{b}}$, respectively. (c-d) The temperature dependence of the inverse susceptibility. Arrows indicate the linear extrapolation in the paramagnetic regime to obtain the Curie temperature.

of the high temperature magnetic susceptibility [Figs. 2(c) and $2(\mathrm{~d})$ ]. The blocking temperature $T_{\mathrm{b}}$, corresponding to the transition from superparamagnetic to the ferrimagnetic blocked state, is obtained from the maximum of the ZFC curve (Table S III [26]). AC susceptometry reveals only a slight temperature dependence of the blocking temperature with AC frequency (Fig. S 4) [26] indicating a well-ordered collinear magnetic state. Further decreasing the temperature, a shallow minimum of the FC magnetization is observed indicating the transition temperature $T_{\mathrm{s}}$ to spin spiral magnetic ordering. The so-called lock-in transition which is reported for bulk material $\left(T_{\text {lock }} \approx 15 \mathrm{~K}\right)$ is not observed for any of our nanocrystalline samples. Comparing the ZFC/FC curves, a shift of Curie, blocking, and spin spiral transition temperatures towards smaller temperatures is clearly visible with decreasing particle size. For a coherent domain size of less than $d_{\mathrm{XRD}}=6.4 \mathrm{~nm}(\mathrm{AA} 400, \mathrm{AA} 350, \mathrm{AA} 300)$, the minima of the FC curve corresponding to the spin spiral magnetic ordering are strongly suppressed. The magnetic phase transitions are also reflected in isothermal magnetization measurements (Fig. $S 5$ [26]). Above the Curie temperature, a linear magnetization dependence is observed corresponding to the paramagnetic state. The SPM state is reflected by a Langevin-shaped magnetization curve. Below $T_{\mathrm{b}}$, hysteresis appears related to the blocked ferrimagnetic order. At the base temperature of $2 \mathrm{~K}$ the magnetization is nonsaturated up to high magnetic fields of $6 \mathrm{~T}$, indicating spin disorder effects in the NPs. This is in line with the significantly different particle sizes observed by PXRD and TEM, where the crystalline part is smaller than the particle size. The structural disorder, related to the lack of crystallinity at the particle surface, is correlated with
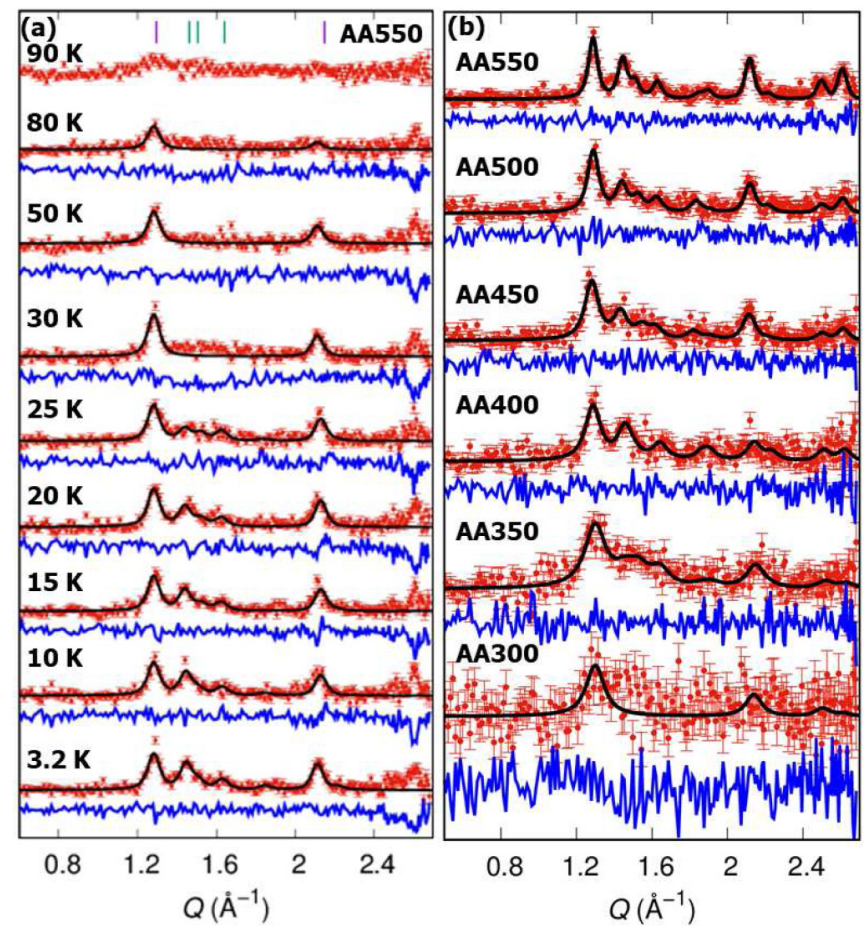

FIG. 3. (a) Temperature dependence of the magnetic scattering cross section (red dots) of cobalt chromite nanoparticles with coherent domain size of $14.0 \mathrm{~nm}$ (sample AA550). Purple and green vertical lines represent the position of fundamental and satellite reflections, respectively. (b) Magnetic scattering contribution of all studied samples recorded at $3.5 \mathrm{~K}$ (red points: experimental data). The black line is Le-Bail fit and the blue line is the residual between the fit and experimental data.

the decrease of the spontaneous magnetization observed with decreasing particle diameter (Table S III [26]).

Neutron diffraction has been employed to investigate the collinear and noncollinear magnetic phases and to resolve the particle size dependence of the magnetic phase transitions in more detail. Polarized neutron diffraction with XYZ polarization analysis gives the opportunity to clearly separate the spin-incoherent, nuclear-coherent, and magnetic scattering contributions (see Fig. S 6 for the complete sample set at $3.5 \mathrm{~K})$. The spin-incoherent diffuse scattering contribution varies with increasing annealing temperature and particle size. We attribute this observation to the different amount of oleic acid surfactant in the sample due to the varying surface to volume ratio and decomposition of the oleic acid starting at $\approx 350^{\circ} \mathrm{C}[11]$.

The magnetic scattering contribution [Fig. 3(a)] directly relates to the magnetic phase structure. At the first magnetic phase transition, from paramagnetic to collinear ferrimagnetic magnetic ordering between 90 to $80 \mathrm{~K}$, fundamental magnetic reflections arise. The transition to noncollinear magnetic ordering around $25 \mathrm{~K}$ is accompanied by additional magnetic satellite reflections. The magnetic reflections at $3.5 \mathrm{~K}$ [Fig. 3(b)] broaden significantly with decreasing coherent domain size (see Fig. S 7 for the complete temperature dependence of the magnetic scattering for all samples). 

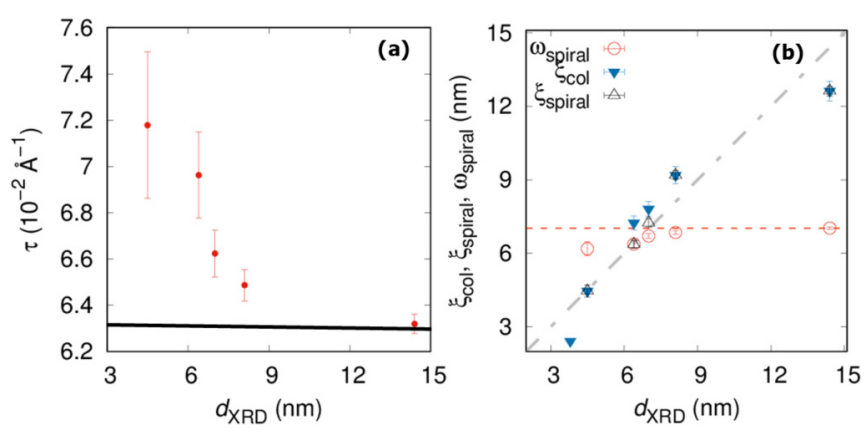

FIG. 4. (a) Size dependence of the propagation vector $\tau$ at the transition temperature (bulk value is presented by black line [13]). (b) Size dependence of the period of spin-spiral $\omega_{\text {spiral }}$, and the coherence length of spin-spiral ordering $\xi_{\text {spiral }}$ and collinear structure $\xi_{\text {col }}$. The grey and red dashed lines represent $\xi=d_{\text {XRD }}$ and the bulk value of $\omega_{\text {spiral }}[10]$, respectively.

The temperature dependence of the integral reflection intensity is obtained from Le-Bail fits of the magnetic scattering cross sections, and is used as magnetic order parameter to determine the magnetic phase boundaries (Figs. S 8 and S 9) [26]. Particle size-dependent magnetic phase transition temperatures are thus accessible with enhanced precision. The ferrimagnetic phase appears between 96(1) to 56(1) K, and the spin-spiral transition temperature changes from $28 \mathrm{~K}$ (similar to bulk [8]) down to $3.5 \mathrm{~K}$ with decreasing particle size (see Table S III [26]).

Based on the satellite reflections in the magnetic neutron diffractograms, the spin spiral propagation vector $(\tau \tau 0)$ was determined using a conical spin spiral model $[27,28]$. In accordance with the method used to obtain the bulk reference [8], the propagation vector was determined for each sample at the corresponding transition temperature $T_{\mathrm{s}}$. The propagation vector component $\tau$ slightly increases with decreasing particle size from $\tau=6.32(1) \cdot 10^{-2}$ to $7.2(3) \cdot 10^{-2} \AA^{-1}$ [see Fig. 4(a)]. Correspondingly, the period of the spin spiral $\left(\omega_{\text {spiral }}=2 \pi / \sqrt{2 \tau^{2}}\right)[$ Fig. 4 (b) $]$ reduces with the particle size. We observe that $\omega_{\text {spiral }}$ approaches the particle size in the range 7 to $6.4 \mathrm{~nm}$, indicating that exactly one period of the spin spiral is compressed slightly to fit into the NP. At smaller particle size of $4.5 \mathrm{~nm}$, the spin spiral phase is still present, suggesting that less than one period may be accommodated within the NP. In the case of the smallest particle size of $3.6 \mathrm{~nm}$, the noncollinear phase is absent. The correlation lengths of the collinear $\left(\xi_{\mathrm{col}}=2 \pi / \mathrm{FWHM}_{\mathrm{col}}\right.$, with FWHM the full-width at half maximum intensity) and noncollinear $\operatorname{order}\left(\xi_{\text {spiral }}=2 \pi / \mathrm{FWHM}_{\text {spiral }}\right)$ are in good agreement with the particle grain size [Fig. 4(b)]. For $d_{\mathrm{XRD}}=3.6 \mathrm{~nm}$ (AA300) only a very broad fundamental magnetic reflection of a collinear magnetic state is observed at $3.5 \mathrm{~K}$. In conjunction with our prior study [13], NPs as small as $2.7 \mathrm{~nm}$ reveal a magnetically frustrated, cluster glass behavior. We associate the diffuse magnetic scattering of the 3.6-nm NPs with the crossover towards a short-range order.

\section{Magnetic phase diagram}

Combining polarized neutron diffraction with macroscopic magnetization results allows deriving the size-induced

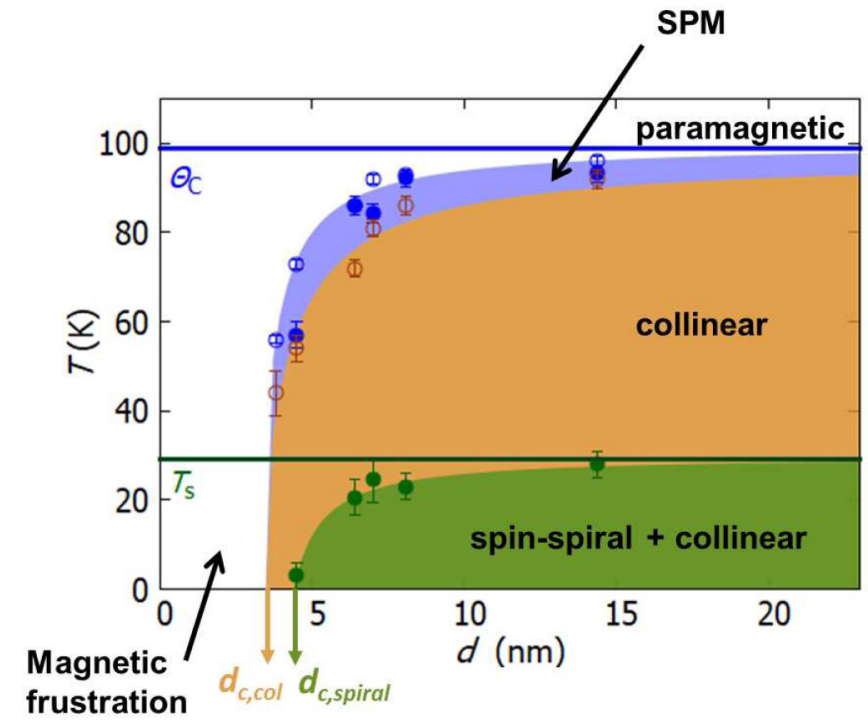

FIG. 5. Size-temperature dependence of the magnetic phase transitions. The blue and green horizontal lines in the magnetic phase diagram correspond to the bulk value of the Curie $\theta_{\mathrm{c}}$ and $T_{\mathrm{s}}$ spin-spiral transition temperature. The Curie $\theta_{\mathrm{c}}$ (blue), blocking $T_{\mathrm{b}}$ (brown) and spin-spiral transition $T_{\mathrm{s}}$ (green) temperatures are described by Eq. (1). $d_{\mathrm{c} \text {,spiral }}$ and $d_{\mathrm{c} \text {,col }}$ denote the critical nanocrystalline size for the spin-spiral and ferrimagnetic ordering, respectively. Results obtained from magnetization and neutron diffraction measurements are described by open and full symbols, respectively.

magnetic phase evolution of nanocrystalline $\mathrm{CoCr}_{2} \mathrm{O}_{4}$ (Fig. 5). We describe the size dependence of the transition temperatures by the following equation [29]:

$$
T(d)=T_{\text {bulk }}\left(1-\frac{C}{d}\right),
$$

where $T_{\text {bulk }}$ is the respective transition temperature of the bulk material [8] and $C=6 \mu(6 M)^{1 / 3} /\left(\rho \pi N_{\mathrm{A}}\right)^{1 / 3} Z^{2 / 3} \pi k^{2}$ with $Z=8$ the formula unit, $k=\sqrt{2} / 4$ the ratio between the atomic radius and lattice parameter of the fcc unit cell, $\mu$ the shape factor, $M$ the molecular weight, $N_{\mathrm{A}}$ the Avogadro constant, and $\rho$ the mass density. All parameters in Eq. (1) were fixed, except the shape factor, which determines the curvature of the phase boundaries. For the bulk value of the blocking temperature, $T_{\mathrm{b}}=94 \mathrm{~K}$ was used, which corresponds to the onset of ferrimagnetic order in polycrystalline $\mathrm{CoCr}_{2} \mathrm{O}_{4}$ [30]. The $1 / d$ dependence in Eq. (1) indicates that the phase transition temperature is determined by the surface-to-volume fraction. We obtain critical particle sizes $\left[T\left(d_{\mathrm{c}}\right)=0\right]$ for the formation of the spin spiral magnetic structure $d_{\mathrm{c} \text {,spiral }}=$ $4.4(1) \mathrm{nm}$, for the collinear magnetic order $d_{\mathrm{c}, \mathrm{col}}=3.3(1) \mathrm{nm}$ and for SPM behavior $d_{\mathrm{c}, \mathrm{spm}}=3.2(5) \mathrm{nm}$. Further reducing the particle size results in magnetic frustration and spin glass behavior as demonstrated for 2.7-nm NPs in our previous work [13].

In addition to the magnetic properties, we find that the spin spiral magnetic order observed by neutron diffraction (see Table S III [26]) is accompanied with the onset of multiferroic behavior evidenced by macroscopic polarization measurements. The appearance of a ferroelectric transition at approximately $28 \mathrm{~K}$ is clearly observed by means of higher 
harmonics measurements of the permittivity $[31,32]$ for the AA500 sample (see Fig. S 10 [26]). The transition temperature is in good agreement with $T_{\mathrm{s}}=23(3) \mathrm{K}$ for this sample. As the NPs have a large surface-to-volume ratio, we expect contact effects to play an important role for direct measurements of the polarization with a high amount of organic surfactant hindering the measurement of a resolvable total macroscopic polarization signal. The incoherent scattering contribution in Fig. S 6 [26] is directly related to the amount of organic material, which varies strongly for each sample, with its lowest contribution in AA500. Considering that the onset of a ferroelectric signal was obtained only for the AA500 sample, we conclude the significant amount of organic material affects the polarization signal strongly for most samples. However, the clear signal obtained for AA500 establishes the multiferroic properties in $\mathrm{CoCr}_{2} \mathrm{O}_{4}$ NPs related to the spin spiral phase.

\section{CONCLUSION}

In summary, the particle size-dependent magnetic phase diagram of nanocrystalline $\mathrm{CoCr}_{2} \mathrm{O}_{4}$ was elucidated by a combination of integral magnetization measurements and polarized neutron diffraction. The continuous decrease of the Curie, blocking, and transition temperatures with nanocrystal size leads to a critical particle size of $d_{\mathrm{c} \text {,spiral }}=4.4(1) \mathrm{nm}$, above which spin spiral magnetic order can exist. A minimum spin spiral period that can be squeezed entirely into the nanoparticle of 6.4(1) $\mathrm{nm}$ has been identified. Below a critical particle size of $d_{\mathrm{c}, \mathrm{col}}=3.3(1) \mathrm{nm}$, collinear magnetism is absent. Instead, the strong inter- and intraparticle interactions arising from structural and surface disorder in the
$\mathrm{CoCr}_{2} \mathrm{O}_{4}$ NPs lead to a frustrated state known as collective superspin glass behavior. Moreover, multiferroic behavior in nanocrystalline $\mathrm{CoCr}_{2} \mathrm{O}_{4}$ is confirmed for the first time. The low content of organic material is presumed crucial for successful dielectric spectroscopy. Our findings have direct consequences for potential applications of cobalt chromite NPs by defining the grain size limits for the presence of spinspiral magnetic structure prerequisite for ferroelectricity in $\mathrm{CoCr}_{2} \mathrm{O}_{4}$. Concerning the multiferroic properties, elimination of the organic residue in the NPs is important to enhance the ferroelectric signal and will pave the way towards the understanding of the coupling of multiferroic behavior and spin spiral magnetic order in $\mathrm{CoCr}_{2} \mathrm{O}_{4} \mathrm{NPs}$.

\section{ACKNOWLEDGMENTS}

We thank Stefan Roitsch for TEM measurements. We thank Alena Klindziuk for her help with data evaluation in the frame of a GIANT internship at ILL in Grenoble, France. This work is based upon experiments performed at the DNS instrument operated by JCNS at the Heinz Maier-Leibnitz Zentrum (MLZ), Garching, Germany. J. Vlček thanks the Czech Science Foundation project no. 18-09347S and support by Ministry of Education, Youth and Sports of the Czech Republic in the frame of project LTC17058 and by COST Action CA15107 MultiComp. Financial support from the German Research Foundation [DFG: Emmy Noether Grant DI 1788/2-1 and Project No. 277146847 SFB 1238 (B02)] as well as the Institutional Strategy of the University of Cologne within the German Excellence Initiative (Max DelbrückPrize for Young Researchers) are gratefully acknowledged. In Memoriam D. Nižňanský.
[1] M. Fiebig, T. Lottermoser, D. Meier, and M. Trassin, Nat. Rev. Mater. 1, 16046 (2016).

[2] T. Kimura, S. Ishihara, H. Shintani, T. Arima, K. T. Takahashi, K. Ishizaka, and Y. Tokura, Phys. Rev. B 68, 060403(R) (2003).

[3] T. Goto, T. Kimura, G. Lawes, A. P. Ramirez, and Y. Tokura, Phys. Rev. Lett. 92, 257201 (2004).

[4] E. Bousquet and A. Cano, J. Phys.: Condens. Matter 28, 123001 (2016).

[5] D. Higashiyama, S. Miyasaka, N. Kida, T. Arima, and Y. Tokura, Phys. Rev. B 70, 174405 (2004).

[6] M. Mostovoy, Phys. Rev. Lett. 96, 067601 (2006).

[7] S. W. Cheong and M. Mostovoy, Nat. Mater. 6, 13 (2007).

[8] Y. Yamasaki, S. Miyasaka, Y. Kaneko, J.-P. He, T. Arima, and Y. Tokura, Phys. Rev. Lett. 96, 207204 (2006).

[9] I. Kim, Y. Seok Oh, Y. Liu, S. Chun, J.-S. Lee, K.-T. Ko, J.-H. Park, J.-H. Chung, and K. H. Kim, Appl. Phys. Lett. 94, 042505 (2009).

[10] L. J. Chang, D. J. Huang, W.-H. Li, S.-W. Cheong, W. Ratcliff, and J. W. Lynn, J. Phys.: Condens. Matter 21, 456008 (2009).

[11] D. Zákutná, A. Repko, I. Matulková, D. Nižňanský, A. Ardu, C. Cannas, A. Mantliková, and J. Vejpravová, J. Nanopart. Res. 16, 2251 (2014).

[12] D. Zákutná, I. Matulková, E. Kentzinger, R. Medlin, Y. Su, K. Nemkovski, S. Disch, J. Vejpravová, and D. Nižňanský, RSC Adv. 6, 107659 (2016).
[13] D. Zákutná, J. Vlček, P. Fitl, K. Nemkovski, D. Honecker, D. Nižňanský, and S. Disch, Phys. Rev. B 98, 064407 (2018).

[14] C. Rath and P. Mohanty, J. Supercond. Novel Magn. 24, 629 (2011).

[15] D. P. Dutta, J. Manjanna, and A. K. Tyagi, J. Appl. Phys. 106, 043915 (2009).

[16] L. Kumar, P. Mohanty, T. Shripathi, and C. Rath, Nanosci. Nanotechnol. Lett. 1, 199 (2009).

[17] Z. Tian, C. Zhu, J. Wang, Z. Xia, Y. Liu, and S. Yuan, J. Magn. Magn. Mater. 377, 176 (2015).

[18] J. K. Galivarapu, D. Kumar, A. Banerjee, and C. Rath, IEEE Trans. Magn. 52, 1 (2016).

[19] J. K. Galivarapu, D. Kumar, A. Banerjee, V. Sathe, G. Aquilanti, and C. Rath, RSC Adv. 6, 63809 (2016).

[20] D. Kumar, J. K. Galivarapu, A. Banerjee, K. S. Nemkovski, Y. Su, and C. Rath, Nanotechnology 27, 175702 (2016).

[21] J. Rodriguez-Carvajal, Physica B 192, 55 (1993).

[22] Y. Su, K. Nemkovskiy, and S. Demirdis, J. Large-Scale Res. Facil. A27 (2015).

[23] W. Schweika and P. Böni, Physica B 297, 155 (2001).

[24] A. Glavic, Plot.py - data plotting and evaluation software, URL https://sourceforge.net/projects/plotpy/.

[25] M.-R. Li, E. McCabe, P. Stephens, M. Croft, L. Collins, S. V. Kalinin, Z. Deng, M. Retuerto, A. S. Gupta, H. Padmanabhan, V. Gopalan, C. P. Grams, J. Hemberger, F. Orlandi, P. Manuel, 
W.-M. Li, C.-Q. Jin, D. Walker, and M. Greenblatt, Nat. Commun. 8, 2037 (2017).

[26] See Supplemental Material at http://link.aps.org/supplemental/ 10.1103/PhysRevB.100.184427 for TEM histograms, XRPD with Le-Bail analysis, AC and DC magnetization measurements, a complete set of neutron diffractograms along with the temperature dependence of the integral intensity of the fundamental (111) and magnetic satellite (111(0)) reflections as well as macroscopic polarization measurements.

[27] J. M. Hastings and L. M. Corliss, Phys. Rev. 126, 556 (1962).
[28] D. H. Lyons, T. A. Kaplan, K. Dwight, and N. Menyuk, Phys. Rev. 126, 540 (1962).

[29] D. Xie, M. Wang, and L. Cao, Phys. Status Solidi B 242, R76 (2005).

[30] G. Lawes, B. Melot, K. Page, C. Ederer, M. A. Hayward, T. Proffen, and R. Seshadri, Phys. Rev. B 74, 024413 (2006).

[31] D. Niermann, C. P. Grams, M. Schalenbach, P. Becker, L. Bohatý, J. Stein, M. Braden, and J. Hemberger, Phys. Rev. B 89, 134412 (2014).

[32] C. Grams, S. Kopatz, D. Brüning, S. Biesenkamp, P. Becker, L. Bohatý, T. Lorenz, and J. Hemberger, Sci. Rep. 9, 4391 (2019). 